\title{
Performance Enhancement of Dynamic Cyclic Shift Code with Direct Detection and AND Subtraction Detection Technique of OCDMA
}

\author{
Tanvi Parikh, Anurag Paliwal \\ GITS Dabok, Udaipur (Raj.) \\ Tanvi.parikh27@gmail.com
}

\begin{abstract}
Optical code division multiple access system (OCDMA) has been important with increasing demands of high capacity and speed for communication in optical networks. Due to OCDMA technique high efficiency is achieved, hence fiber bandwidth is fully used .In this paper we will focus on DCS (Dynamic cyclic shift) code with their different detection technique using optiwave system design tool. Due to this codes we will eliminated MAI (multiple access interference) and improve BER (bit error rate), PIIN( Phase induced intensity noise) and make orthogonality between users in the system. We will use two different detection technique i.e Direct detection technique and AND subtraction detection technique to implement the codes with different parameters. In this paper, the study of different parameters is based on conference a paper that is mainly demonstrated on an experiment to Enhancement a performance of DCS (Dynamic cyclic shift) code with there different parameter of OCDMA using optiwave system design tool.
\end{abstract}

Keywords: OCDMA (optical CDMA), BER (Bit error rate), MAI (Multiple access interference),DCS (Dynamic cyclic shift), PIIN( Phase induced intensity noise)

\section{Introduction}

Optical CDMA is providing each user asynchronous access to network without wavelength control. OCDMA system has been recognized as one of the most important technologies that many user can share media simultaneously and increase transmission capacity of an optical fiber. OCDMA has already been employed for LAN and for different network access application. But OCDMA suffers from different noise such as shot noise , thermal noise, dark current and phase induced intensity noise (PIIN), Multiple access interference (MAI). Due to these many Codes are introduced such as FCC (Flexible cross correlation), DCS (Dynamic cyclic shift), MD (multi diagonal), KS, Modified Frequency Hopping (MFH), Modified Quadratic Congruence (MQC) and Modified Double Weight (MDW) codes. However, these codes have several limitations such as the code is either too long (e.g. Optical Orthogonal Code and Prime Code), construction is complicated (e.g. MFH code), or poorer cross correlation and fixed an even natural number for Modified Double Weight (MDW) code. But to avoid such interferences and make technology more efficient we will use these Codes. . In these paper we will discuss about DCS code with there different parameter such as Optical fiber length, No of users, Weight, Receiver load resistor, Receiver noise temperature, Data bit rate, Operating Wavelength, Electrical bandwidth, Broadband effective power, Quantum efficiency of Detector using optiwave system design tool. Due to this codes we will eliminated MAI (multiple access interference) and improve BER (bit error rate), PIIN( Phase induced intensity noise) and make orthogonality between users in the system .We will use two different technique i.e Direct detection technique and AND subtraction detection technique to implement these codes with different parameters. In this paper 
there are 6 section: 1) Introduction 2) Construction of DCS code 3) System performance analysis 4) Numerical and simulation analysis 5) Conclusion 6) References.

\section{Construction of DCS Code}

We have developed a new code referred to as DCS, which includes the parameters $N$, $W$ and $\lambda c$, where $N$ denotes the code length (i.e., the number of total chips), $W$ the code weight, and $\lambda c$ indicates the in-phase cross-correlation. The cross-correlation $\lambda c$ between any pair of code sequences must be small enough. This property would ensure that each code sequence can be easily distinguished from every other address sequence. In other words, we seek to make the MAI which remains insignificant when compared to the energy contained in the information received. For code sequences

$X=(x 1, x 2, \ldots x N)$ and $Y=(y 1, y 2, \ldots \ldots . . . y N)$, the cross-correlation is given by

$$
\lambda c=\sum_{i=1}^{N} x i y i
$$

The codes with ideal in-phase cross-correlation $(\lambda c \leq 1)$ are required in the OCDMA systems since these codes eliminate multi-user interference and suppress the effect of the PIIN. The technique utilised for constructing the DCS code is detailed further below.

\subsection{Algorithm for DCS Code Design}

The new code family suggested here is represented as $\left(\mathrm{N}=\sum_{i=1}^{W-1} 2^{\mathrm{i}}+\mathrm{D}, \mathrm{W}, \lambda_{\mathrm{c}}\right)$ where $\mathrm{i}=(0,1, \ldots \ldots \mathrm{W}-1)$, denotes a positive integer number and $D$ represents the dynamic part. The next steps are followed to construct the DCS code words:

Step 1

First we construct a sequence $S^{i}$ of integer numbers that are elements of the Galois field $\mathrm{GF}(N)=\{1,2, \ldots \ldots, N\}$ over an integer number $N$, using the expression

$$
S^{i}=\quad \begin{gathered}
\left(2^{i}\right)(\bmod N), i=0,1 \\
\left(S^{i-1}+2^{i}\right)(\bmod N), i=2,3 \ldots \ldots W-1
\end{gathered}
$$

Here $\mathrm{S}^{\mathrm{i}}, \mathrm{N}$, and $\mathrm{W}$ are the elements over the Galois field $\mathrm{GF}(\mathrm{N})$.

Step 2

After that we construct a sequence $T i$ of binary numbers $(0,1)$ basing on the generated sequence $S i$ and using the mapping method

$$
\mathrm{T}_{\mathrm{i}}=\left\{\begin{array}{cc}
1 & \text { for } S i \\
0 & \text { otherwise }
\end{array}\right.
$$

Step 3

Now we combine the binary sequence of each $T i$ that has been generated in the step 2, to get

the first code sequence of the first user. The elements of the first code word are given by

$\mathrm{C}_{1}=\mathrm{T}_{0}+\mathrm{T}_{1}+\mathrm{T}_{2} \ldots \ldots \ldots \ldots \mathrm{T}_{\mathrm{w}-1}$

The method for producing the binary sequence $(0,1)$ based on $\mathrm{GF}(\mathrm{N})$ is shown in Figure 1. We have also illustrated generation of the first DCS code word sequence in Table 1 for the codeparameters $\mathrm{W}=3, \mathrm{D}=8$ and $\mathrm{N}=22$ as an example. In Table $1, \mathrm{C}_{1}$ denotes the first code word of the DCS code. In Fig. 2.1.1 we display a procedure of combining the sequences Ti needed for generating the first code word of the DCS code. 
Table 2.1.1. Generation of sequence $\mathrm{Si}$ and binary sequence $\mathrm{Ti}$ for the case of $W=4, D=8$ and $N=22$.

\begin{tabular}{|c|c|c|}
\hline $\mathrm{i}$ & $\mathrm{S}^{\mathrm{i}}$ & $\mathrm{T}_{\mathrm{i}}$ \\
\hline 0 & 1 & 10000000000000000000 \\
\hline 1 & 2 & 01000000000000000000 \\
\hline 2 & 6 & 00000100000000000000 \\
\hline 3 & 14 & 11000100000001000000 \\
\hline $\mathrm{C}_{1}$ & $\mathrm{C}_{1}$ & 11000100000001000000 \\
\hline
\end{tabular}

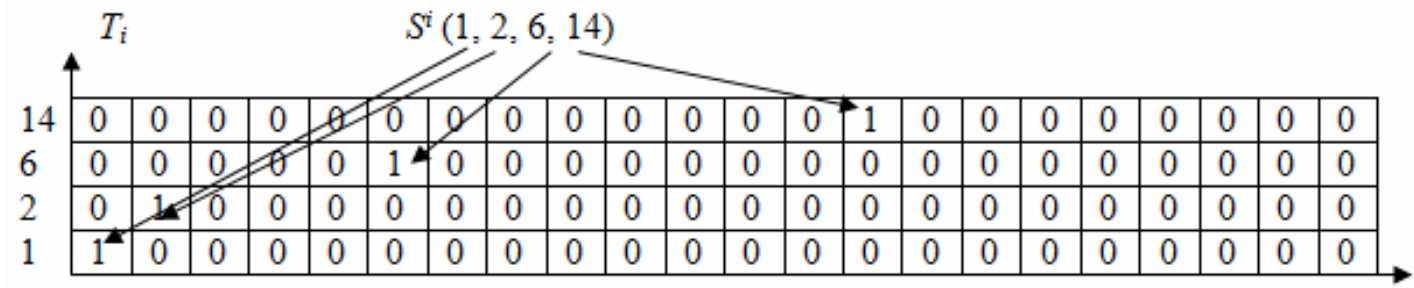

Figure 2.1.1. Generation of sequence Ti for the case of $N=22, D=8$ and $W=$ 4

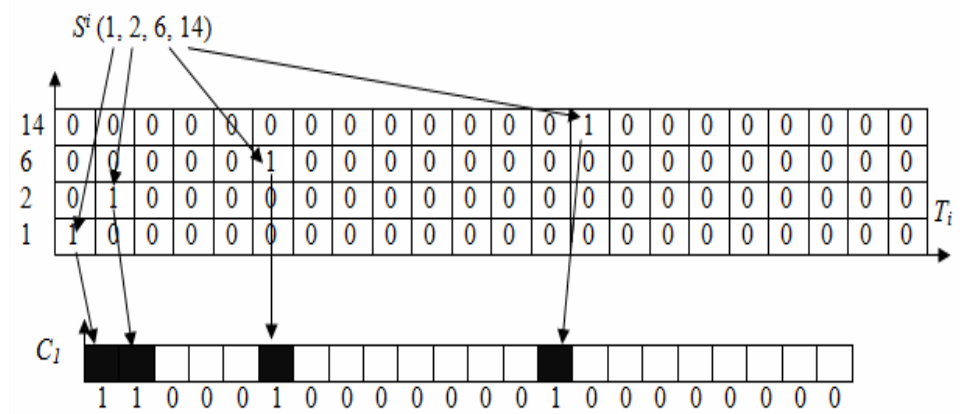

Figure 2.1.2. Combination of sequences Ti used for producing the first code word

After generating the first code sequence, we use a cyclic shift method to produce the code sequence for the rest of the users. There are $N-1$ different sequences which can be generated from the first code sequence. The number of the code sequences is equal to the code length $(K=N)$. In order to achieve the cyclic shift property with a minimum crosscorrelation ( $\lambda c \leq 1)$, we always consider the $D$ values given by the inequality $D>7$. Otherwise, the cross-correlation value would have been larger than one. In our code, the number of users can be increased by increasing the dynamic sequence $D$ only, while the weight sequence part remains unchanged. An example is presented in Table 2 for $K=14$ subscribers, where $W=3$ and $D=8$.

Table 2.1.2 shows the weight $W$, the code length $N$, and the cross-correlation $\lambda c$ for 30 users. It is clearly seen that the DCS code can be generated with lower code weights ( $W$ $=2$ ) and less lengths, when compared to the other codes. It is also seen that the DCS code reveals better crosscorrelation values ( $\lambda c \leq 1)$. Furthermore, if the chip width (i.e., the filter bandwidth) of $0.8 \mathrm{~nm}$ is used, the spectral widths required for the OOC, RD, MDW, MQC, KS, MFH and DCS codes are 291.2, 28, 44.8, 72, 115.2, 33.6 and $24 \mathrm{~nm}$, respectively. As evident from Table 2.1.2, there is no other code that can be generated with the code weight equal to two, except of the DCS code 
Table 2.1.2 DCS code words for the case of $W=3, D=8$ and $K=14$.

\begin{tabular}{|c|}
\hline Suscriber code word \\
\hline 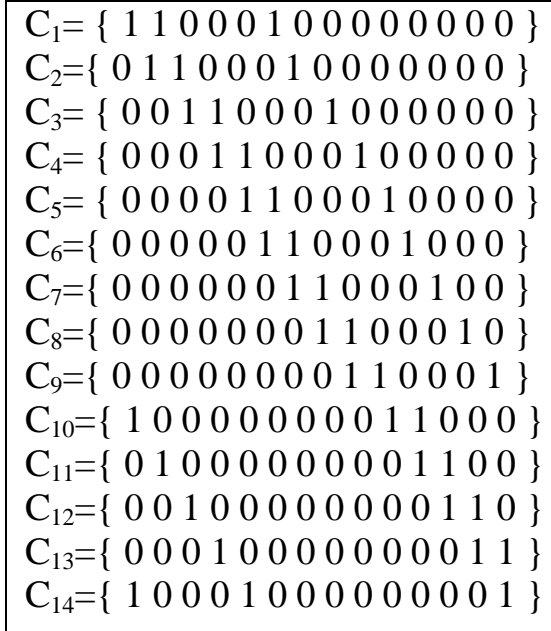 \\
\hline
\end{tabular}

\section{3) System Performance Analysis}

\subsection{Direct Detection Technique for DCS Code}

The Direct subtraction technique has been used as detection method. This technique is fully capable of eliminating the MAI, reducing complexity of receiver and improving performance of the system. In the frame of this technique the direct detection technique, has one pair of decoder and detector is required compared to other techniques that required two branches of inputs to the receiver like these in complementary subtraction techniques. There is also no subtraction process involved. This is achievable for the simple reason that the information is assumed to be adequately recoverable for any of the chips that do not overlap any other chips from other code sequences, since DCS code is designed with no overlapping chips. Thus the detector will only need to filter through the clean chips (no overlapping chips) to be directly detected by the photo diode as normal intensity modulation with the direct detection scheme. The MAI effect has been successfully and completely been eliminated because only the required signal spectra in the optical domain will be filtered. Furthermore, it is important to note that the whole spectrum still needs to be transmitted to maintain the address signature. This distinguishes the technique from Wavelength Division Multiplexing (WDM) technology 


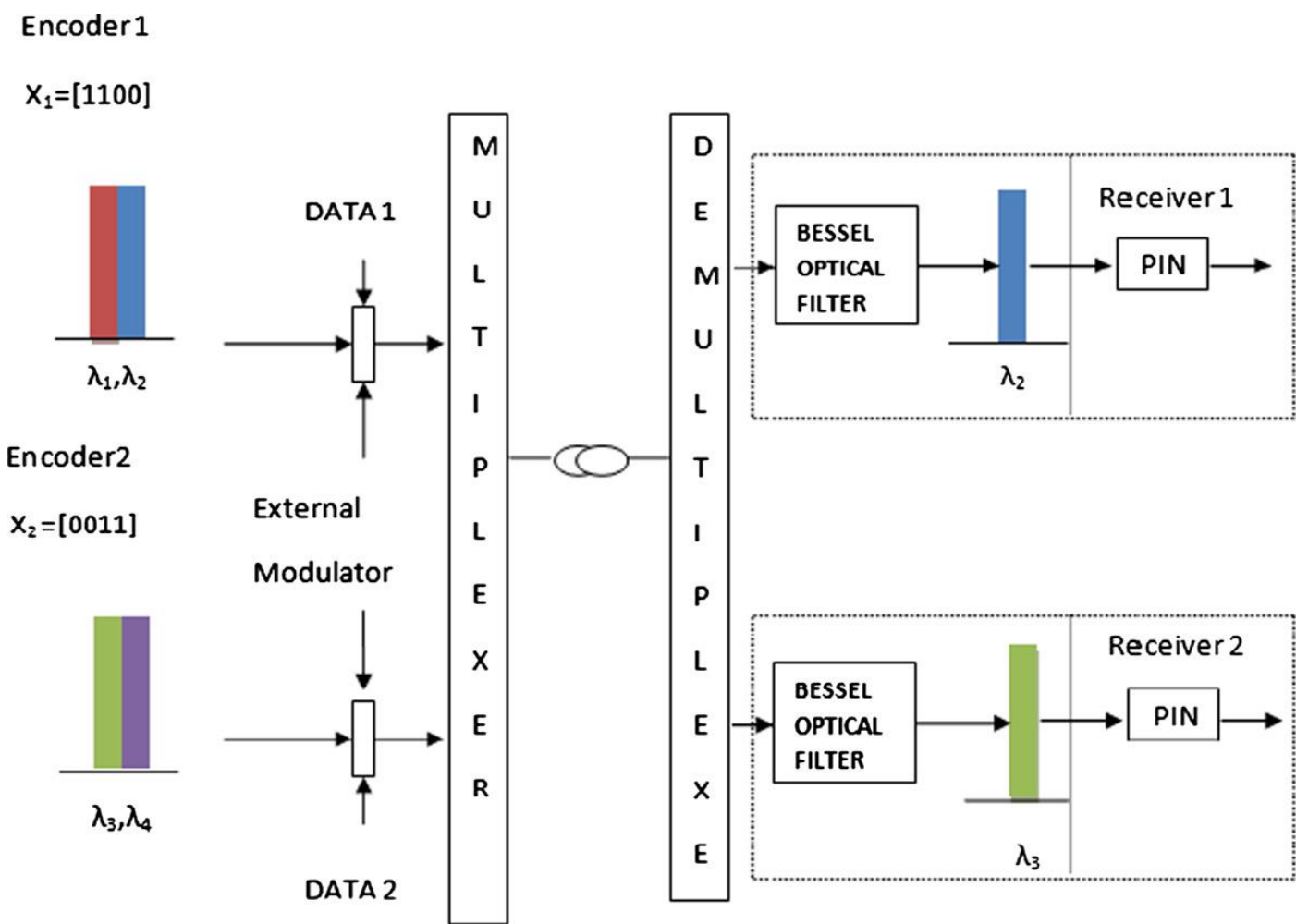

Figure 1. Direct Detection Technique for DCS Code

\subsection{AND subtraction Detection Technique for DCS}

The AND subtraction technique has been used as a detection method. This technique is fully capable of eliminating the MAI, reducing complexity of receiver and improving performance of the system. In the frame of this technique, a spectral amplitude signal at the receiver side is split into two branches. The upper branch is the signal for a user $\mathrm{X}$ associated with cross-correlation between $\mathrm{X}$ and $\mathrm{Y}$, and the lower branch should be a cross-correlation result from the AND operation between $\mathrm{X}$ and $\mathrm{Y}$, which have the same cross-correlation magnitude associated with the signal in the upper branch

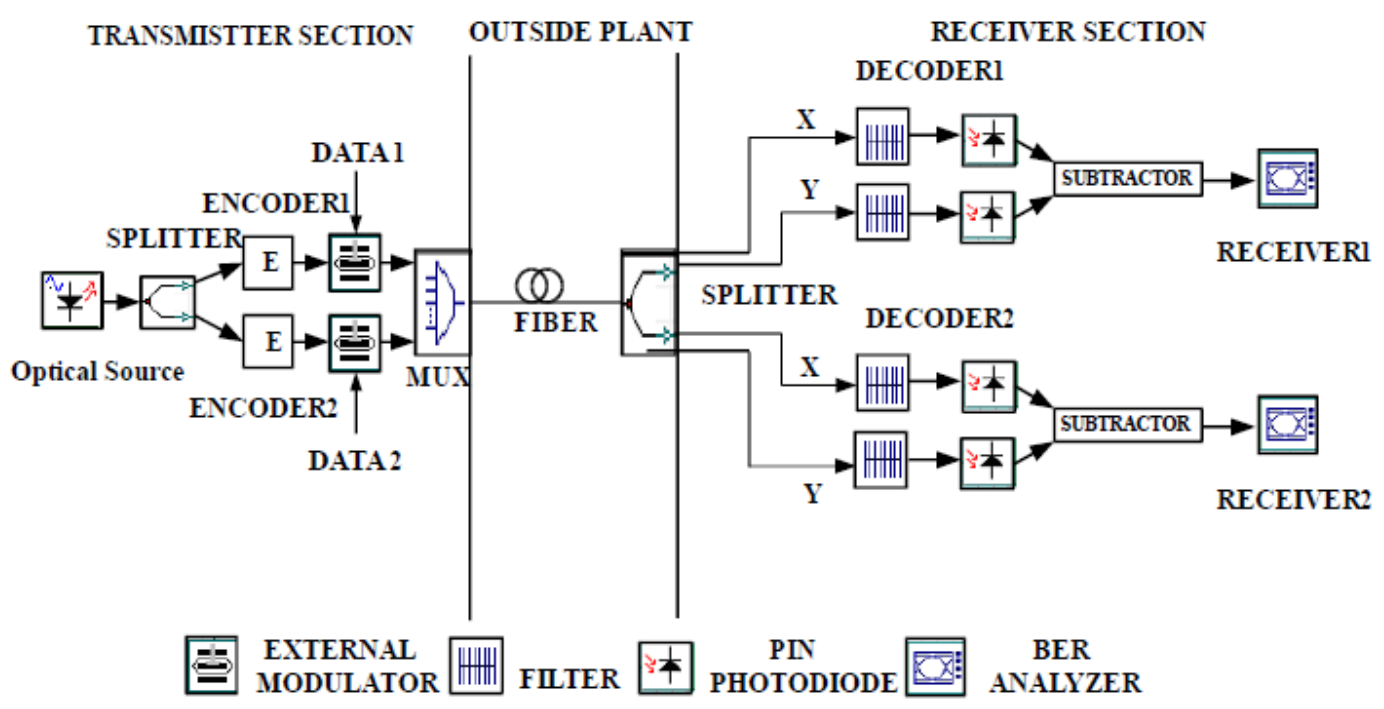

Figure 2. AND Subtraction Technique for DCS Code 


\section{Numerical and Simulation Analysis}

\subsection{Numerical Analysis}

The performance of the DCS code has been compared numerically with the recently suggested codes such as the KS code, the EDW code, the MFH code, FCC . We evaluate the BER and the SNR using
$\mathrm{SNR}={\frac{\mathrm{I}}{\sigma^{2}}}^{2}=$
$\left\{R \operatorname{Psr}(\mathrm{W}-1) /{ }_{N}\right\}^{2}$

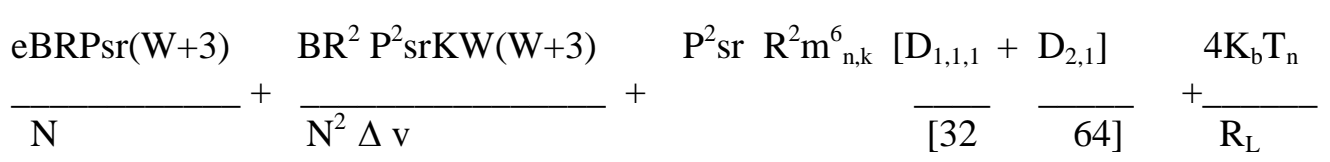

Here $D_{1,1,1}$ is the three-tone third-order inter-modulation at $f i+f K-f l$, and $D_{2,1}$

represents the two-tone third-order intermodulation at $2 f i-f K$

$$
B E R=\frac{1}{2} \operatorname{erfc}(\sqrt{S N R / 8})
$$

Typical parameters used in our numerical analysis and calculations

\begin{tabular}{|l|l|l|}
\hline Symbol & Parameter & Value \\
\hline$H$ & $\begin{array}{l}\text { Quantum efficiency of photo- } \\
\text { detector }\end{array}$ & 0.6 \\
\hline $\mathrm{P}_{\mathrm{sr}}$ & Broadband effective power & $-10 \mathrm{dBm}$ \\
\hline $\mathrm{B}$ & Electrical bandwidth & $80 \mathrm{MHz}$ \\
\hline$\lambda_{\mathrm{o}}$ & Operating wavelength & $1550 \mathrm{~nm}$ \\
\hline $\mathrm{R}_{\mathrm{b}}$ & Data bit rate & $155 \mathrm{M} \mathrm{bit} / \mathrm{s}$ \\
\hline $\mathrm{T}_{\mathrm{n}}$ & Receiver noise temperature & $300 \mathrm{~K}$ \\
\hline $\mathrm{R}_{\mathrm{L}}$ & Receiver load resistor & $1030 \Omega$ \\
\hline
\end{tabular}

The relationship between the number of simultaneous users and the BER for the DCS code having $\mathrm{W}=4$. It is clearly seen that the performance of the DCS code is much higher by AND detection technique. The maximum acceptable BER is 10-9 that we achieved by the DCS code compared. We can ascertain from this fact that the DCS code has a small length, the number of the active users is equal to the code size and the cross-correlation is $\lambda c=1$ or $\lambda c=0$,

\section{2. Simulation Analysis}

The hybrid system has been simulated using the software "OptiSimTM", The simulation is implemented for the two-subcarrier channel basing on the DCS code. Here we adopt the data rate $155 \mathrm{Mbit} / \mathrm{s}$ for each subcarrier channel. Furthermore, the subcarrier frequencies are set to be equal or larger than two times (the Niquest frequency) the bit rate. Each optical channel has the spectral width of $0.8 \mathrm{~nm}$. The simulation has been carried out for a standard single-mode optical fibre ITU-T G.652. All the parameters that describe the attenuation $(0.25 \mathrm{~dB} / \mathrm{km})$, the dispersion $(18 \mathrm{ps} /(\mathrm{nm}$ per $\mathrm{km})$, and the nonlinear effects (four-wave mixing and self-phase modulation), have been activated and specified according to their typical industrial values, in order to simulate a real 
environment as close as possible. The noise generated at the receivers has been set to be random and totally uncorrelated. The dark current value has been put to be $5 \mathrm{nA}$. We have also used the thermal noise coefficient $1.8 \times 10-23 \mathrm{~W} / \mathrm{Hz}$ for each of the photodetectors. The performance of the system has been characterised by referring to the BER and the eye diagram pattern. The effect of fibre distance on the performance of our system having two subcarrier frequencies for different light source powers. It is clear that the dispersion has significant impact on the system performance when the fibre length increases. Our simulation results indicate that the system performance is deteriorating by about more than one order of magnitude, whenever the dispersion effect is activated in the simulation model. In addition, our results testify that the system performance is worsening as the fibre length increases from 20 to $50 \mathrm{~km}$. The fig shows the PIIN for DCS code will eliminated MAI(multiple access interference) and improve BER (bit error rate), PIIN( Phase induced intensity noise) and make orthogonality between users in the system.

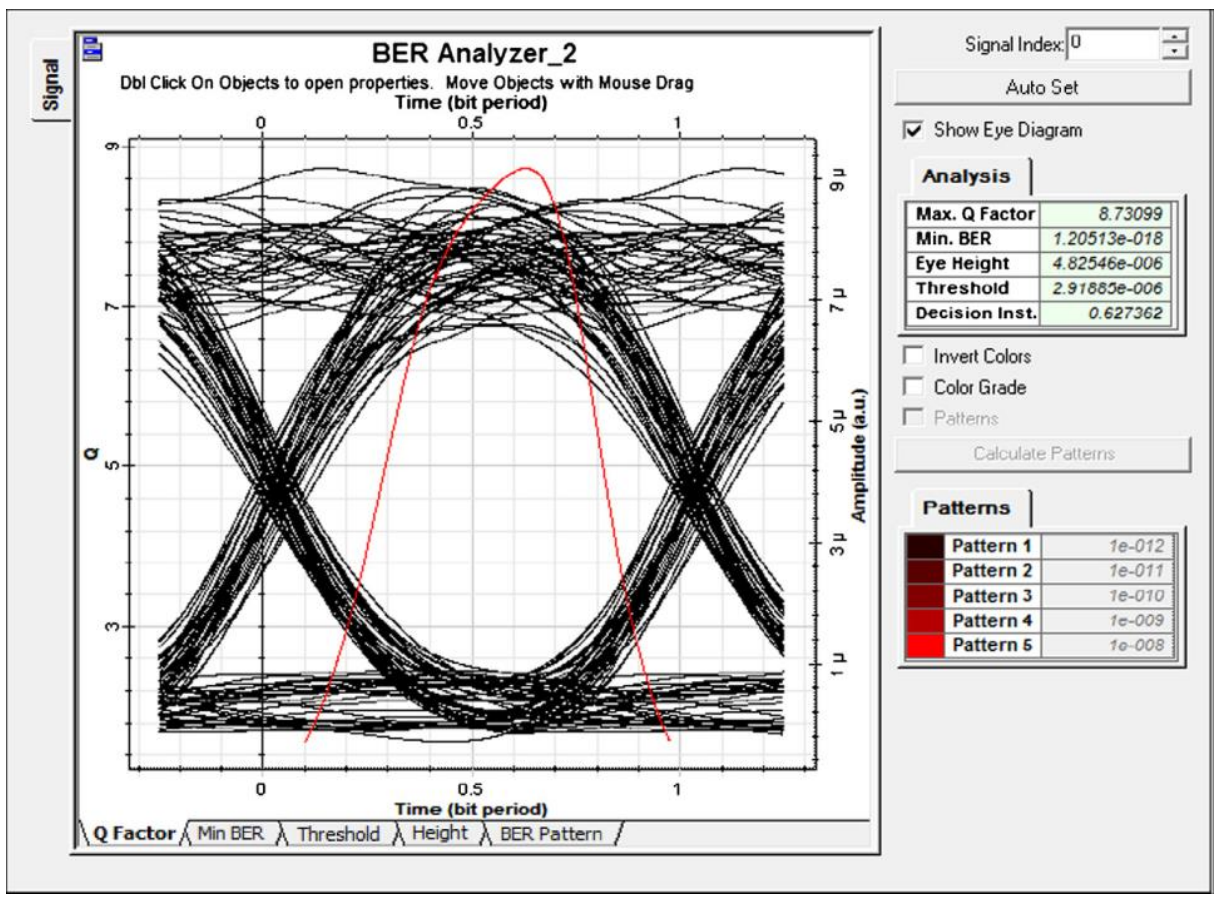

\section{Conclusion}

The system degradation due to PIIN can be suppressed using DCS code, results in enhancing BER performance. The proposed DCS code is robust in term of received power, Psr as well as a reliable number of simultaneous users. The performance of the proposed DCS code achieves high cardinality (number of simultaneous users) code. The performance of the system is revealed to improve significantly, because the total loss is reduced as the AND detection technique requires less number of filters in the decoder. In addition, making use of the code words with less crosscorrelation value mitigates the PIIN, which improves the overall system performance. It suppresses the MAI, as compared to the system that uses the other SAC codes, and enables carrying large numbers of code words and subcarrier channels.

\section{References}

[1] Khaleghi F and Kavehrad M, 1995. A subcarrier multiplexed CDM optical local area network, theory and experiment. IEEE Trans. Commun. 43: 75-87. 
[2] Yin H, Liang W, Ma L and Qin L, 2009. A new family of two-dimensional triple-code weight asymmetric optical orthogonal code for OCDMA networks. Chinese Opt. Lett. 7: 102-105.

[3] Biao C, Fu-chang W, Jian-dong H and Sai-ling H, 2005. A novel OCDMA drop unit based on fiber gratings and 2D wavelength-time codes. Optoelectronics Lett. 1: 53-56.

[4] Prucnal P R, Santoro M A and Fan T R, 1986. Spread spectrum fiber-optic local area network using optical processing. J. Lightwave Technol. 4: 547-554.

[5] 890-892.

[6] T.H. Abd, S.A. Aljunid, Hilal A. Fadhil, R.B. Ahmad, and M.A. Rashid, "New Approach For Evaluation of The Performance of Spectral Amplitude Coding-Optical Code Division Multiple Access System on High-Speed Data

[7] Rate", IET Communications, Vol. 6, No. 12, 2012, pp. 1742-1749.

[8] M.S. Anuar, S.A. Aljunid, R.Badlishah, N.M. Saad, and I. Andonovic, "Performance Analysis of Optical Zero Cross Correlation in OCDMA System”, Journal of Applied Sciences, Vol. 7, No. 23, 2007, pp. 3819-3822.

[9] H.M.R. Al-Khafaji, S.A. Aljunid, and Hilal A. Fadhil, "Spectral Efficiency of Unipolar SACOCDMA System Considering Noise Effects", Proceedings of IEEE Symposium on Industrial Electronics and Applications, (Langkawi), September, 25-28, 2011, pp. 218 - 222.

[10] Hilal A. Fadhil, S. A. Aljunid, and R.B. Ahmed, "A Effective Design of Optical Code-Division Multiple Access Network Using Random Diagonal Codes", Proceedings of 6th National Conference on Telecommunication Technologies and 2nd Malaysia Conference onPhotonics, (Putrajaya), Aug, 26-28, 2008 , 